\title{
Papers
}

\section{Postmortem findings after fatal anaphylactic reactions}

\author{
Richard S H Pumphrey, Ian S D Roberts
}

\begin{abstract}
Aims-To determine the frequency at which classic manifestations of anaphylaxis are present at necropsy after fatal anaphylactic reactions.

Methods-A register has been established of fatal anaphylactic reactions in the UK since 1992, traced from the certified cause of death and other sources. Details of the previous medical history and the reaction suggest anaphylaxis as the cause of death for 130 cases; a postmortem report was available for 56 .

Results-The 56 deaths studied included 19 reactions to bee or wasp venom, 16 to foods, and 21 to drugs or contrast media. Death occurred within one hour of anaphylaxis in 39 cases. Macroscopic findings included signs of asthma (mucous plugging and/or hyperinflated lungs) (15 of 56), petechial haemorrhages (10 of 56), pharyngeal/laryngeal oedema (23 of 56), but for 23 of 56 there was nothing indicative of an allergic death. Mast cell tryptase was raised in 14 of 16 cases tested; three of three tested had detectable IgE specific for the suspected allergen.

Conclusions-In many cases of fatal anaphylaxis no specific macroscopic findings are present at postmortem examination. This reflects the rapidity and mode of death, which is often the result of shock rather than asphyxia. Investigations that might help determine whether anaphylaxis was the cause of death had rarely been performed. In the presence of a typical clinical history, absence of postmortem findings does not exclude the diagnosis of anaphylaxis.

(f Clin Pathol 2000;53:273-276)
\end{abstract}

Keywords: necropsy; anaphylaxis; asthma

Laboratory Medicine Academic Group,

University of

Manchester, Oxford

Road, Manchester

M13 9PT, UK

I S D Roberts

Correspondence to:

Dr Pumphrey

email:

Richard.Pumphrey@man.ac.uk

Accepted for publication 11 August 1999

Acute allergic reaction is an uncommon but well recognised cause of sudden death. Anaphylactic reactions can cause variable combinations of symptoms including generalised flushing, urticaria, angio-oedema, vomiting, diarrhoea, conjunctivitis, rhinorrhoea, sneezing, and coughing; there may be loss of consciousness as a result of shock, or breathing difficulty caused by increased upper or lower airways resistance.
More severe reactions can lead to respiratory or cardiac arrest. Asphyxia can follow upper airways obstruction as a result of pharyngeal or laryngeal oedema, or lower airways obstruction as a result of bronchospasm, in some cases with mucous plugging. Cardiac arrest can follow respiratory arrest, or can occur without respiratory difficulty, as a result of either direct effects of mediators of anaphylaxis on the heart, or profound shock resulting from peripheral vasodilatation, often combined with angiooedema causing loss of intravascular fluid. In cases where shock is established within minutes of the start of the reaction, there may be no time for other features to appear.

In a study of fatal anaphylaxis we encountered cases in which, despite a convincing clinical history, anaphylaxis had been rejected as a cause of death because of the lack of specific findings at necropsy. There have been no previous studies to establish the spectrum of postmortem findings in anaphylactic death from all causes. Our study reports the first 56 necropsies that were reported to the register of fatal anaphylactic reactions.

\section{Methods}

A register has been established of all fatal anaphylactic reactions in the UK since 1992. Cases were identified from the Office of National Statistics (ONS) database (1992-8). Since 1993, all entries on this database have been coded, allowing searches for anaphylaxis as a cause of death. Before this date, searches were performed by looking for text strings that might indicate that anaphylaxis contributed to the cause of death. Requests for measurement of mast cell tryptase in serum after reactions had been sent to a number of UK laboratories; these were used to identify further fatal reactions. Other notifications came from the Anaphylaxis Campaign, the police, pathologists, and members of the public.

Within limitations imposed by the local
ONS, it was possible to retrieve detailed information about the fatal reactions from the coroners and medical staff involved in the care of individuals on the register. The total number of cases so far registered is 169 , and details of previous medical history, the reaction, and its treatment are known for 152. A cause other 
than anaphylaxis seemed more likely to have caused death in 22 when full details of the reaction were known. Of the remaining 130 cases, 37 were reactions to food, 32 to venom, one hydatid, and 60 to iatrogenic allergens. The mode of death was respiratory failure as a result of airway obstruction in 57, shock in 39, and a combination of the two in 34 cases. Adrenaline was administered in 17 before arrest and in 65 during resuscitation.

Necropsies were performed in most cases and we have had access to reports in 56 cases to date. In all cases with a postmortem report available, necropsy was performed on the instruction of the coroner. Of these, $83 \%$ were performed by NHS pathologists and $17 \%$ by forensic pathologists. The data presented in our study refer to these 56 cases.

\section{Results}

CLINICAL HISTORY

The clinical variables are summarised in table 1. Of the 56 cases studied, 19 were reactions to bee or wasp venom, 16 reactions to food allergens, and 21 to drugs. In all cases, there was a history of collapse after contact with allergen. An asthmatic component was documented in 23 cases. Death occurred within one hour of anaphylaxis in 39 cases (immediate deaths) and was delayed in 17 cases (median, 60 hours; range, 3-720 hours). In 28 cases, there was a history of previous allergic reactions. For food related deaths the allergens were nuts (14), prawns (1), and nectarines (1). Iatrogenic reactions included eight reactions to antibiotics, 10 to anaesthetic/induction agents, one to acetazolamide, one to technetium, and one to contrast medium (Hexabrix).

The mode of death was not the same for each cause. All food allergic reactions caused difficulty in breathing that led to respiratory arrest in 13 of 16 cases, whereas shock without difficulty in breathing was more common in venom (eight of 19) and iatrogenic reactions (12 of 21).

Forty nine of 56 deaths were given a postmortem diagnosis of anaphylaxis and five of asthma (two of these were certified as caused by anaphylaxis after inquest). One death that followed the first dose of amoxycillin was diagnosed as being caused by chronic bronchitis and emphysema, but was accepted as being the result of anaphylaxis after the finding of a high mast cell tryptase concentration. The remaining case is given in detail because it illustrates the difficulty ascertaining the incidence of anaphylaxis from death certificates.
A 76 year old woman with a history of asthma and bronchitis was prescribed a course of amoxycillin by her general practitioner for a chest infection. Within 30 minutes of taking the first dose she became unwell with difficulty breathing; she collapsed unconscious but was resuscitated by a paramedic.

After two weeks recovery in hospital she was given a test dose of amoxycillin orally because there had been doubt as to the cause of her collapse. Within 15 minutes of this test she developed difficulty breathing and collapsed unconscious. She was given treatment for an anaphylactic reaction and recovered.

After three weeks convalescence, she was prescribed cefaclor by her general practitioner for persistent symptoms of infected bronchitis. By now she had become suspicious of antibiotics and delayed taking the first dose until she was at home, accompanied by a friend. At this time, apart from her usual productive cough, she was well. Within five minutes of taking the first dose, she became ill, collapsed, and died. The chemical structures of cefaclor and amoxycillin are very similar in that part of the molecule recognised by allergic responses, and crossreactivity is recognised.

There were no findings thought to be indicative of anaphylaxis at necropsy and no blood was taken for further investigation. Death was certified as the result of acute tracheobronchitis.

\section{POSTMORTEM FINDINGS}

Table 1 summarises the findings at necropsy in each of the three groups. The most common reported finding was non-specific pulmonary congestion and oedema, which was present in $41(73 \%)$ cases. Features suggesting an allergic reaction were present in $59 \%$ of all cases (table 1) and in $100 \%, 67 \%$, and $37 \%$ of immediate deaths in the food, venom, and iatrogenic groups, respectively. Cutaneous erythema/ urticaria was identified in only three necropsies; laryngeal and/or pharyngeal oedema in 23 ( $8 \%$ and $49 \%$ of immediate deaths, respectively). Upper airway oedema was more common in deaths related to food allergens ( $77 \%$ of immediate deaths) than in those after reactions to venom or drugs $(40 \%$ and $30 \%$ of immediate deaths, respectively). Hyperinflation of the lungs and/or mucous plugging of airways, suggesting an asthmatic component, were noted in 15 cases, 13 of which were immediate deaths. The presence of petechial haemorrhages, also suggesting an asphyxial component to the death, was found in 10 cases, eight of which were immediate deaths. Brain

Table 1 Summary of clinical and postmortem findings

\begin{tabular}{|c|c|c|c|c|}
\hline & $\begin{array}{l}\text { Group } 1 \\
\text { (venom) }\end{array}$ & Group 2 (food) & $\begin{array}{l}\text { Group } 3 \\
\text { (drugs) }\end{array}$ & Total \\
\hline Male:female & $11: 8$ & $5: 11$ & $6: 15$ & $22: 34$ \\
\hline Age in years, median (range) & $64(28-85)$ & $20(13-53)$ & $63(7-76)$ & $52(7-85)$ \\
\hline Minutes from contact to anaphylaxis, median (range) & $20(5-120)$ & $30(17-127)$ & $5(1-120)$ & $20(1-127)$ \\
\hline Immediate deaths & 16 & 13 & 10 & 39 \\
\hline Delayed deaths & 3 & 3 & 11 & 17 \\
\hline Cutaneous erythema/oedema & 1 & 2 & 0 & 3 \\
\hline Upper airway oedema & 6 & 10 & 7 & 23 \\
\hline Mucous plugging of bronchi and/or hyperinflation & 7 & 5 & 3 & 15 \\
\hline Petechial haemorrhages & 4 & 5 & 1 & 10 \\
\hline Pulmonary congestion and/or oedema & 14 & 9 & 18 & 41 \\
\hline
\end{tabular}


swelling suggestive of cerebral hypoxia was noted in 15 cases, of which seven were delayed deaths. In food allergic deaths, the presence of food in the stomach was noted in 10 necropsies, but the known allergen (nuts) was positively identified in only one. Other important pathologies identified at necropsy were ischaemic heart disease in four cases, emphysema in one, and carcinoma of the breast in two.

Histology was performed in 20 necropsies. In two cases that showed upper airway oedema, histology of laryngeal mucosa demonstrated a pronounced eosinophilia. Histology of the bronchi demonstrated mucosal oedema, inflammation with eosinophilia, and epithelial sloughing in three of four cases with mucous plugging identified macroscopically. Serum concentrations of mast cell tryptase were documented in only 16 necropsies. Of these, concentrations were raised in 14 . In the two cases in which tryptase was not raised, death was delayed by eight and 480 hours. In the first of these, the assay was performed on postmortem serum, in the latter an antemortem sample was analysed, but the precise timing of the sample was not documented. Specific IgE had been measured in five cases and was positive in three; the two negative results were not to the same allergen as that supposed to have caused the reaction.

\section{Discussion}

Ours is the first study to report postmortem findings in an unselected series of fatal anaphylactic reactions from all causes. It was undertaken because of repeated instances of alternative postmortem diagnosis in cases of typical anaphylactic death that had been encountered in maintaining the UK register of fatal anaphylactic reactions. A median of 20 fatal anaphylactic reactions has been identified each year since the register was started in 1992. Approximately half of these were iatrogenic and a quarter each caused by foods or insect venom. At the time of our study, clinical details were known for 152 fatal reactions on the register. Thirty four cases, despite strongly suggestive clinical histories, did not have anaphylaxis identified as a cause on the death certificate. Twenty two subjects did have anaphylaxis listed in the causes of death, but were thought to have died from a reason other than anaphylaxis after assessment of all the available evidence. The 56 cases reported here are representative of the 130 deaths thought to be caused by anaphylactic or anaphylactoid reactions.

We found that 23 of the 56 anaphylactic deaths studied had no macroscopic postmortem findings suggestive of anaphylaxis. Because the chance of identifying deaths as anaphylactic is greater when recognisable features are present at necropsy, the true fraction of deaths with negative findings is likely to be higher. Other than single case reports, ${ }^{2-7}$ there has been no previous systematic study of postmortem findings after anaphylactic deaths from all causes. Mosbech reported the features of 26 fatal insect stings including brief reference to the postmortem findings. ${ }^{8}$ It was noted that local reactions were often not evident and, when present, were small, reflecting the rapidity of death. Pre-existing ischaemic heart disease, which might have contributed to cardiovascular collapse, was found in five of his cases, and two deaths may have been caused by asphyxia as a result of stings in the mouth. None of the subjects who died of venom related deaths in our series had heart disease and, although three had been stung in the mouth, they died more rapidly than the subjects that Mosbech studied. Two had a high concentration of mast cell tryptase, suggesting that death was caused by anaphylaxis rather than local swelling from the sting. In a study of 43 fatal drug related reactions, Delage and colleagues ${ }^{9}$ reported upper airway oedema and bronchial obstruction to be present in only $22 \%$ and $25 \%$ of cases, respectively.

It can be difficult to establish the cause of a reaction even when it is not fatal. Factors that suggest anaphylaxis include an immediately preceding challenge with an allergen known to cause reactions, clinical features of an allergic reaction, a previous history of reactions to similar or crossreactive allergens, specific IgE antibodies to the allergen thought to have caused the reaction, and measurable products of a reaction such as urinary methyl histamine or serum mast cell tryptase. In the case of fatal reactions, skin prick tests are not possible, but $\mathrm{IgE}$ is stable in serum for several weeks and can be measured in postmortem serum. ${ }^{10}$ Specific $\operatorname{IgE}$ can be measured to venoms and many foods, including all those commonly causing anaphylaxis. IgE can also be measured to some penicillins and a thiocholine epitope that in many cases crossreacts with muscle relaxants, which are possibly the most common cause of fatal iatrogenic anaphylaxis. These tests had been performed in only three of 56 cases, all with positive results, with two additional cases tested for a possibly crossreactive allergen with negative results.

Mastocytosis might predispose to reactions similar to anaphylaxis that might not require the presence of IgE antibodies. Two cases on the register were noted to have systemic mastocytosis, one dying after a bee sting, the other, previously reported, ${ }^{7}$ during an anaesthetic. This latter case is included in those reported here.

Mast cell $\beta$-tryptase is a protein that is released from mast cell granules during anaphylactic reactions. $\alpha$-Tryptase is a similar protein that is secreted by resting mast cells and is raised in mastocytosis. The tests for mast cell tryptase available in the UK before 1997 detected $\beta$-tryptase and, since then, the most widely used test does not distinguish between $\alpha$-tryptase and $\beta$-tryptase. ${ }^{11}{ }^{12} \beta$-Tryptase is reasonably stable in serum over a period of days and, therefore, can be measured in postmortem samples. ${ }^{13} 14$ There has been discussion about the value of these tests in assisting the determination of the cause of death because $\beta$-tryptase has been found to be raised in non-anaphylactic fatalities ${ }^{14}$ and to be within the normal range in asthmatic deaths. ${ }^{16}$ 
There is no doubt, however, that tryptase is raised after anaphylactic reactions and particularly reactions that cause shock - the very cases that might leave little else to show their cause. ${ }^{17}$ Tryptase had been measured in 16 of the 56 cases here, and was raised in 14. In the two negative cases, death was delayed by at least eight hours, by which time previously raised values might have returned to normal; after peak concentrations are achieved in the first few hours of a reaction, serum tryptase values fall, with a half life of approximately two hours. ${ }^{13}$ Personal communication with pathologists indicates that the most common reason for not performing IgE and tryptase measurements is ignorance that these tests are available.

The case history reported here is similar to others we have encountered where the lack of specific findings prevented the postmortem conclusions from including anaphylaxis as a cause of death. In one of these following the first dose of cefuroxime, tryptase was found to be raised after the inquest; anaphylaxis was then accepted as the cause of death but the certified cause (bronchopneumonia) was not amended. Another was thought to have been the result of asthma until evidence was discovered at inquest that the fatal asthma attack followed within minutes of the first dose of a course of cephalexin in a patient with a previous history of penicillin allergy.

In five of 16 deaths caused by nut allergy, the postmortem cause of death was given as asthma, despite other features of a generalised reaction noted in the clinical record. It is possible that many other acute asthma deaths in those with a history of food allergy might have been reactions to food. A recent study from Sweden has identified soy proteins as a possible cause of such reactions. ${ }^{18}$ This raises the question of which deaths should be classified as anaphylactic, and which asthmatic. When a wasp sting causes sudden death as a result of asthma, this will commonly be recorded as being caused by anaphylaxis. The argument is that anaphylactic reactions are acute, severe, generalised allergic reactions; a reaction in the lungs resulting from a sting on the foot must be part of a generalised reaction. Asthmatic deaths resulting from inhaled allergen could be the result of a local reaction, but those from food, like those from stings, must be part of a generalised reaction and should all be classified as being caused by anaphylaxis. This is surely the case if there have been any other features of a generalised reaction such as flushing, urticaria, angioedema, or vomiting-features that are very often recorded but may leave no changes detectable at necropsy. More consistent certification of these deaths as anaphylaxis would certainly improve documentation of cases and facilitate the monitoring of the true frequency of fatal reactions.

We conclude that in many cases of fatal anaphylaxis no specific findings are present at necropsy. This is particularly likely after reactions that caused rapid onset of shock. In the presence of a typical history, the absence of specific findings at necropsy does not exclude the diagnosis of anaphylaxis. Tests for specific IgE and mast cell tryptase might help to determine whether anaphylaxis is the cause of death, but are not often used. We recommend that they should be performed in all cases with a suggestive clinical history and no specific findings at necropsy. Furthermore, the possibility of anaphylaxis should be considered in all cases of sudden unexpected death with absent postmortem findings; femoral vein blood should be sampled, and the separated serum frozen, to enable later testing for specific IgE antibodies and mast cell tryptase. The results of these assays should be interpreted in the context of clinical history and postmortem findings. Because interpretation is often difficult, specialist immunological advice should be sought. Features in the clinical history suggesting a generalised reaction should be considered when deciding between asthma and anaphylaxis as the cause of death. Venom or food related asthmatic deaths should be classified as the result of anaphylaxis because the asthma was part of a generalised allergic reaction.

We thank the Anaphylaxis Campaign for early notification of many fatalities, and all coroners, pathologists, and others who have helped collect this data.

1 Adkinson NF, Jr. Beta-lactam crossreactivity. Clin Exp Allergy 1998;28(suppl 4):37-40.

2 Patel F. Seafood-induced fatal anaphylaxis. Med Sci Law 1998;38:354-7.

3 Ansari MQ, Zamora JL, Lipscomb MF. Postmortem diagnosis of acute anaphylaxis by serum tryptase analysis. A case report. Am f Clin Pathol 1993;99:101-3.

4 Ciesielski-Carlucci C, Leong P, Jacobs C. Case report of anaphylaxis from cisplatin/paclitaxel and a review of their hypersensitivity reaction profiles. Am $\mathcal{F}$ Clin Oncol 1997;20: 373-5.

5 Belton AL, Chira T. Fatal anaphylactic reaction to hair dye. Am f Forensic Med Pathol 1997;18:290-2.

6 Prahlow-JA, Barnard-JJ. Fatal anaphylaxis due to fire ant stings. Am f Forensic Med Pathol 1998;19:137-42.

7 Vaughn STA, Jones GN. Systemic mastocytosis presenting as profound cardiovascular collapse during anaesthesia. Anaesthesia 1998;53:804-9.

8 Mosbech H. Death caused by wasp and bee stings in Denmark 1960-1980. Allergy 1983;38:195-200.

9 Delage C, Irey NS. Anaphylactic deaths: a clinicopathologic study of 43 cases. $\mathcal{F}$ Forensic Sci 1972;17:525-40.

10 Yunginger JW, Nelson DR, Squillace DL, et al. Laboratory investigation of deaths due to anaphylaxis. $\mathcal{F}$ Forensic Sci 1991;36:857-65.

11 Schwartz LB, Sakai K, Bradford TR, et al. The alpha form of human tryptase is the predominant type present in blood at baseline in normal subjects and is elevated in those with systemic mastocytosis. F Clin Invest 1995;96:2702-10.

12 Hogan AD, Schwartz LB. Markers of mast cell degranulation. Methods: A Companion to Methods in Enzymology tion. Methods: $A$.

13 Schwartz LB, Yunginger JW, Miller J, et al. Time course of appearance and disappearance of human mast cell tryptase in the circulation after anaphylaxis. F Clin Invest 1989;83: 1551-5.

14 Edston E, van Hage-Hamsten M. $\beta$-Tryptase measurements post-mortem in anaphylactic deaths and controls. Forensic Sci Int 1998;93:135-42.

15 Randall B, Butts J, Halsey JF. Elevated postmortem tryptase in the absence of anaphylaxis. F Forensic Sci 1995;40:208 11.

16 Salkie ML, Mitchell I, Revers CW, et al. Postmortem serum levels of tryptase and total and specific IgE in fatal asthma. Allergy Asthma Proc 1998;19:131-3.

17 Schwartz-HJ, Yunginger-JW, Schwartz-LB. Is unrecognized Schwartz-HJ, Yunginger-JW, Schwartz-LB. Is unrecognized
anaphylaxis a cause of sudden unexpected death? Clin Exp allergy 1995;25:866-70.

18 Foucard T, Malmheden Yman I. A study of severe food reactions in Sweden-is soy protein an underestimated cause of food anaphylaxis? Allergy 1999;54:261-5. 